wenn man annehmen wollte, dafs der von uns benutzte Campher auch ein Gemenge von homologen Verbindungen gewesen sei.

Göttingen, den 29. September 1867.

Ueber die Cyanverbindungen des Mangans; von James H. Eaton und Rud. Fittig.

\title{
I. Mangancyanür-Cyankalium.
}

Ueber das Verhalten der Manganoxydullösungen gegen Cyankalium sind sehr verschiedene, zum Theil einander widersprechende Angaben gemacht worden. Nach $\mathrm{Haidlen}$ und Fresenius*) entsteht auf Zusatz von wenig Manganoxydullösung zu einer Lösung von Cyankalium zuerst eine hell rothbraune Flüssigkeit, dann auf Zusatz von mehr Manganlōsung ein schmutzig rothgelber Niederschlag von Mangancyanür, der sich in sehr viel Cyankalium zu einer rothbraunen Flüssigkeit auflöst, welche an der Luft unter $A b-$ scheidung von Manganoxydhydrat zersetzt wird. Rammelsberg**) fand, dafs der Niederschlag von Mangancyanür schwer zu untersuchen sei, weil er an der Luft augenblicklich braun werde, dafs er sich in Cyankalium nur theilweise zu einer blafsgelben Flüssigkeit auflöse, welche bei der geringsten Erwärmung unter Abscheidung von Manganoxydhydrat roth werde und dann beim Verdunsten kleine rothe Krystalle von der Zusammensetzung $3 \mathrm{KCy}+\mathrm{Mn}_{2} \mathrm{Cy}_{3}$ gebe. Nach

*) Diese Aunalen XLIII, 129.

**) Pogg. Anu. XLII, 117. 
Balard*) endlich soll der Nicderschlag, den Cyankalium in einer Manganoxydullösung bewirkt, im Ueberschufs von Cyankalium sich nicht merklich auflōsen, aber an der Luf sich färben und dann in Cyankalium löslich werden. Aus dieser letzteren Lōsung sollen sich nach dem Verdunsten lange rothe Krystallnadeln abscheiden.

Nach der übereinstimmenden Annahme von $\mathrm{Haidlen}$ uud Fresenius, Rammelsberg und Gmelin enthält die Lösung des zuerst entstehenden Niederschlags in überschüssigem Cyankalium ein dem gelben Blutlaugensalz analoges Mangandoppelcyanür, aber es gelang keinem dieser Chemiker, dasselbe aus seiner Lōsung abzuscheiden.

Unsere Beobachtungen weichen nicht unwesentlich von allen früheren Angaben ab. Fügt man zu einer wässerigen Lòsung von Cyankalium **) tropfenweise eine mäfsig concentrirte neutrale Lösung von essigsaurem Mangan, so scheidet sich zuerst ein schmutzig hellgelber Niederschlag ab, der sich, wenn die Cyankaliumlösung concentrirt ist, beim Umschütteln jedesmal sofort wieder auflöst. Ist die Cyankaliumlösung aber verdünnt, so kann man denselben abfiltriren; aber er ist so unbeständig, dafs er sich selbst mit ausgekochtem Wasser nicht ohne Zersetzung auswaschen läfst. Dabei färbt er sich äufserlich braun und im Innern grün. Wir haben diese Verbindung in reinem Zustande nicht erhalten können; mehrere Versuche machen es indefs wahrscheinlich, dafs sie nicht das isolirte Mangancyanür ist, sondern noch Kalium in chemischer Verbindung enthält.

Fährt man mit dem tropfenweisen Zusatz der Manganlösung unter öfterem Umschütteln fort, so entsteht bald ein

*) Compt. rend. 1844, 909.

*) Wir benutzten zu allen unseren Versuchen das gowöhnliche, nach Liebig's Methode bereitete, käufliche Cyanhalium. 
voluminöser grüner Niederschlag, der sich beim Schütteln nicht mehr auflöst. Dieser kann gleich nach der Darstellung abfiltrirt und mit ausgekochtem Wasser leicht ohne Zersetzung ausgewaschen werden. Er verändert sich dann auch bei mehrtăgigem Stehen über Schwefelsäure nicht und kann in trockenem Zustande auf $100^{\circ}$ erhitzt werden, ohne dafs er seine grüne Farbe verliert oder sein Gewicht ändert. Lãfst man den Niederschlag aber längere Zeit in der Flüssigkeit stehen, aus welcher er ausgefällt wurde, so färbt er sich braun.

Das ũber Schwefelsãure getrocknete Salz ist wasserfrei und hat die Zusammensetzung $\mathrm{HnCy}_{2}+\mathrm{KCy}$.

Zur Analyse wurde die getrocknete Verbindung mit Schwefelsäure eingedampft, der Rückstand in Wasser gelöst, mit Schwefelammonium ausgefällt und das Schwefelmangan nach der Methode von Rose durch Glühen mit Schwefel im Wasserstoffstrome bestimmt. Die rom Schwefelmangan abfiltrirte Flüssigkeit wurde verdunstet und das Kalium als schwefelsaures Salz gewogen. Diesclbe Methode wurde auch bei den nachher zu beschreibenden Salzen angewandt. Sie ergab uns stets ein gutes Resultat.

0,6219 Grm. des grünen Salzes lieferten 0,3163 HnB $=0,19996$ Mn und $0,3185 \mathrm{~K}_{8} \mathrm{~S} \theta_{4}=0,1429 \mathrm{~K}$.

\begin{tabular}{cccc} 
& \multicolumn{2}{c}{ Berechnet } & Gefunden \\
$\overbrace{\mathbf{H} v}$ & 55 & 31,96 & 32,15 \\
$\mathrm{~K}$ & 39,1 & 22,72 & 22,98 \\
$3(G \mathrm{~N})$ & 78 & 45,32 & - \\
\cline { 2 - 3 } & 172,1 & 100,00 &
\end{tabular}

Diese Verbindung hat demnach eine analoge Zusammensetzung, wie das bei der Darstellung der Blausäure zurückbleibende weifse, an der Luft aber rasch blau werdende Salz, aus welchem wahrscheinlich auch der Niederschlag besteht, den eine Eisenoxydullösung mit Blutlaugensalz erzeugt. In überschüssigem Cyankalium löst sich diese grüne 
Manganverbindung leicht und vollständig zu einer schwach gelb gefärbten Flüssigkeit, welche auf Zusatz von Alkohol einen hellblauen, aus kleinen glänzenden Krystallblāttchen bestehenden Niederschlag abscheidet, der anfänglich sehr voluminōs ist, sich aber bald zu Boden setzt und dann abfiltrirt und mit einem Gemisch von gleichen Theilen Alkohol und Wasser ohne Zersetzung ausgewaschen werden kann. Aus Wasser lärst sich diese Verbindung nicht umkrystallisiren; löst man sie aber bei gewöhnlicher Temperatur oder bei gelinder Wärme in mõglichst wenig Cyankaliumlösung auf und setzt die schwach gelb gefärbte Lösung an einen kühlen Ort, so scheiden sich sehr bald prachtvolle, tiefblaue, völlig durchsichtige, von einander isolirte und sehr regelmäfsig ausgebildete quadratische Tafeln $a b$, die wir mehrmals in der Gröfse von mehreren Quadratmillimetern erhielten.

Dasselbe Salz bildet sich auch ohne Alkoholzusatz, wenn man zu einer concentrirten Cyankaliumlösung so viel der essigsauren Manganlösung setzt, dafs eben ein bleibender Niederschlag entsteht, dann rasch filtrirt und die Lösung an einen kühlen Ort stellt. In beliebig grofser Quantität aber läfst sich das Salz dadurch erhalten, dal's man in eine concentrirte Lösung von essigsaurem Mangan Stücke festen Cyankaliums legt und von Zeit zu Zeit einige Tropfen Wasser hinzufügt. Es scheidet sich dann zuerst die grüne Verbindung ab, aber allmälig verschwindet diese wieder und es bildet sich an der Oberfläche des Cyankaliums eine Kruste von tiefblauen Krystallen. Durch sorgfältiges Reguliren des Mengenverhältnisses der Manganlōsung, des Cyankaliums und des $W$ assers lässt sich auf diese $W$ eise fast alles vorhandene Mangan in die blaue Verbindung überführen.

Es ist auffällig, dafs die Lösungen, aus denen sich dieses tiefblaue Salz abscheidet, selbst in concentrirtem $\mathrm{Zu}$ - 
stande nahezu farblos oder höchstens schwach gelb gefärbt sind.

Das Salz enthält Krystallwasser, welches es an der Luft und namentlich über Schwefelsäure sehr rasch verliert. Schon nach 24 stündigem Stehen über Schwefelsäure ist es vollständig wasserfrei, hat dann aber seine blaue Farbe verloren und ist undurchsichtig grau violett geworden. In Berührung mit Cyankaliumlösung kehrt die ursprüngliche blaue Farbe sofort zurück.

Das über Schwefelsãure oder bei $100^{\circ}$ getrocknete Salz hat die Zusammensetzung $\mathrm{MnCy}_{2}+4 \mathrm{KCy}$.

1. $0,6479 \mathrm{Grm}$. der grofsen blauen Krystalle, die 24 Stunden über Schwefelsäure gestanden hatten, verloren bei $100^{\circ}$ nicht an Gewicht und lieferten auf die oben beschriebone Weise analysirt 0,14782 Ins $=0,09345$ und $0,61425 \mathrm{~K}_{2} 8 \theta_{4}=$ $0,2757 \mathrm{~K}$.

1I. $0,45375 \mathrm{Grm}$. des mit Alkohol erbaltenen und bei $100^{\circ}$ getrockneten blauen Niederschiages gaben 0,10539 M $8=0,06663 \mathrm{Mn}$.

\begin{tabular}{|c|c|c|c|c|}
\hline \multirow[b]{2}{*}{ Mn } & \multicolumn{2}{|c|}{ Berechnet } & \multicolumn{2}{|c|}{ Gefunden } \\
\hline & 55 & 14,97 & $\begin{array}{c}1 . \\
14,42\end{array}$ & $\begin{array}{c}\text { II. } \\
14,68\end{array}$ \\
\hline $4 \mathrm{~K}$ & 156,4 & 42,56 & 42,55 & - \\
\hline $6(\theta N)$ & $\frac{156}{367,4}$ & $\begin{array}{r}42,47 \\
10000\end{array}$ & - & - \\
\hline
\end{tabular}

Die Krystallwasserbestimmung ist mit einigen Schwierigkeiten verbunden, weil das Salz so aufserordentlich leicht verwittert. Wir haben die aus der Lösung herausgenommenen grofsen Krystalle unit Fliefspapier von der anhängenden Mutterlauge möglichst befreit, dann mit verdünntem Alkohol abgewaschen, hierauf wieder mit Papier abgetrocknet und sie endlich noch kurze Zeit einem Luftstrom von 30 bis $40^{\circ}$ ausgesetzt. Alle diese Operationen wurden sehr rasch nach einander ausgeführt.

I. 0,2115 Grm. der so belandelten Krystalle verloren beim Erhitzen auf $100^{\circ} 0,0265 \mathrm{H}_{\mathbf{z}} \theta$. 
II. $0,28887 \mathrm{Grm}$, verloren $0,03662 \mathrm{H}_{z} \theta$.

$$
\begin{aligned}
& \overbrace{\text { Berechnet }}^{\text {Gefunden }} \\
& \left.\begin{array}{rcrrrr}
\mathrm{M} \mathrm{nCy}_{2}+4 . \mathrm{KCy} & 367,4 & 87,19 & & - & - \\
3 \mathrm{H}_{2} \mathrm{O} & 54 & 12,81 & & 12,53 & 12,68 \\
& & 421,4 & 100,00
\end{array}\right]
\end{aligned}
$$

Das Mangancyanür-Cyankalium ist demnach nach der Formel $\mathrm{HnCy}_{2}+4 \mathrm{KCy}+3 \mathrm{H}_{2} \theta$ zusammengesetzt. Diese Formel entspricht vollständig der des gelben Blutlaugensalzes $\mathrm{FeCy}_{\mathbf{z}}+\mathbf{4} \mathrm{KCy}+3 \mathrm{H}_{\mathbf{z}} \boldsymbol{\theta}$, mit welchem das Salz auch isomorph zu sein scheint.

Sehr merkwürdig ist das Verhalten des MangancyanürCyankaliums gegen Wasser. Die blauen Krystalle lösen sich in Wasser von gewöhnlicher Temperatur leicht zu einer ganz klaren, fast farblosen Flüssigkeit auf, aber nach-kurzem Stehen trübt sich diese Lösung von selbst, und nach und nach scheidet sich daraus ein grüner Niederschlag ab, der sich mit ausgekochtem Wasser auswaschen, neben Schwefelsäure trocknen und auf $100^{\prime \prime}$ erhitzen läfst, ohne sich $\mathrm{zu}$ zersetzen.

0,3966 Grm. der so erhaltenen Verbindung lieferten 0,20721 $\mathrm{MnS}$ $=0,13099 \mathrm{Mn}$ und $0,19728 \mathrm{~K}_{2} \mathrm{~s} \theta_{4}=0,08856 \mathrm{~K}$.

\begin{tabular}{cccc} 
& \multicolumn{2}{c}{ Berechnot } & Gefunden \\
Mfn & 55 & $\frac{31,96}{32}$ & 33,03 \\
K & 39,1 & 22,72 & 22,33 \\
$3(G N)$ & 78 & 45,32 & -
\end{tabular}

Der Niederschlag hat demnach dieselbe Zusammensetzung, wie der oben beschriebene, direct aus Cyankalium und essigsaurem Mangan erhaltene (S. 159), und auch in seinen Eigenschaften stimmt er vollständig mit demselben überein. Das Mangancyanür-Cyankalium zersetzt sich also in wässeriger Lösung freiwillig nach der Gleichung :

$\left(\mathrm{HCO}_{2}+4 \mathrm{KCy}\right)=\left(\mathrm{MnCy}_{2}+\mathrm{KCy}_{2}\right)+3 \mathrm{KCy}$.

Die Hälfte des in dem Salze enthaltenen Cyans spaltet 
sich als Cyankalium ab, und diese Zersetzung ist vollständig analog derjenigen, welche das gelbe Blutlaugensalz beim Erwärmen mit verdünnter Schwefelsäure erleidet, wobei bekanntlich ebenfalls nur die Hälfte des vorhandenen Cyans als Blausäure entwickelt wird und cin Salz von analoger Zusammensetzung zurückbleibt.

Siedendes Wasser zersetzt das Mangancyanür-Cyankalium sofort unter Abscheidung von Manganoxydhydrat. Kalter Alkohol löst und zersetzt das Salz anfänglich nicht, aber nach einigen Tagen wird es oberflächlich oxydirt und braun; heifser Alkohol zersetzt es rascher, aber doch viel langsamer, als Wasser. Bei Gegenwart von Cyankalium wird das Salz auch durch Wasser nur langsam zersetzt. Die Lösung bleibt klar, aber fügt man nach mehreren Tagen Alkohol zu derselben, so bildet sich kein blauer Niederschlag mehr, sondern ein rother von Mangancyanid-Cyankalium. Aus diesem Grunde darf man auch die aus Cyankaliumlösung erhaltenen grofsen blauen Krystalle nicht zu lange in der Mutterlauge liegen lassen, denn nach 1 bis 2 Tagen schon lösen sie sich wieder vollständig auf und man erhält sie dann auch bei sehr starker Abkühlung nicht wieder.

In einem Kohlenwasserstoff lassen sich die blauen Krystalle unverändert aufbewahren.

Beim Erhitzen auf $100^{\circ}$ wird das trockene Salz nicht verändert, aber bei $200^{\circ}$ zersetzt es sich und verwandelt sich in eine braune Masse.

Die frisch bereitete wässerige Lösung des reinen Mangancyanür-Cyankaliums giebt mit Chlorbaryum, Chlorcalciun, essigsaurer Thonerde und salpetersaurem Uranoxyd keine Niederschläge, mit essigsaurem Manganoxydul einen hellbraunen Niederschlag, mit oxydfreiem Eisenvitriol sowohl wie mit Eisenchlorid dunkelblaue, dem Berlinerblau ähnliche Niederschläge, mit Chlorkobalt einen purpurbraunen, mit 
Chlornickel einen graulichen, mit essigsaurem Blei einen gelben, mit Zinnchlorür einen apfelgrünen, mit Quecksilberchlorid und salpetersaurem Silber einen schmutzig-weifsen und mit Goldchlorid einen dunkelbraunen Niederschlag. Platinchlorid wird durch die Lösung des Salzes nicht gefällt, aber nach einiger Zeit scheidet sich Kaliumplatinchlorid ab. Die meisten dieser Niederschläge sind leicht veränderlich und lassen sich nicht ohne Zersetzung mit Wasser auswaschen. Wir haben bei mehreren derselben vergeblich versucht, sie in einem zur Analyse geeigneten Zustand zu erhalten. Es ist daher schwer zu sagen, ob sie eine constante und analoge Zusammensetzung haben, wie die durch Blutlaugensalz bewirkten Niederschläge, oder ob sie nur Gemenge sind. Die krystallinische Beschaffenheit einiger derselben aber scheint auf eine bestimmte chemische Verbindung hinzudeuten.

Salzsäure scheidet aus der frisch bereiteten Lösung des blauen Salzes einen schmutzig-weilsen, leicht zersetzbaren, im Ueberschufs der Säure leicht löslichen Niederschlag ab. Schwefelalkalien fällen Schwefelmangan, aber erst nach einiger Zeit.

\section{Mangancyanid-Cyankalium .}

Wenn man die blauen Krystalle des Mangancyanür-Cyankaliums einige Tage in der cyankaliumhaltigen Mutterlauge an der Luft stehen läfst, so lösen sie sich auf und gehen ohne Abscheidung von Manganoxydhydrat in MangancyanidCyankalium über. Dieselbe Zersetzung findet statt, wenn man die aus der Lōsung herausgenommenen Krystalle, ohne sie vorher abzuwaschen, an der Luft liegen lärst; denn die kleine Menge der anhaftenden Mutterlauge reicht hin, um diese Veränderung zu bewirken. Sie färben sich dann roth und lösen sich nachher in Wasser zu einer rothen Flüssigkeit klar auf. Sehr rasch, aber unter Abscheidung von Man- 
ganoxydhydrat, erfolgt diese Umwandlung, wenn man die Lösung des blauen Salzes zum Sieden erhitzt oder im Wasserbade auf ein geringes Volumen verdunstet. Im letzteren Falle scheidet sich eine rothe Masse ab, welche sich unter Zurücklassung von Manganoxydhydrat in Wasser mit intensiv blutrother Farbe auflosst. Versetzt man diese Lösung mit etwas Cyankalium und läfst sie neben Schwefelsäure freiwillig verdunsten, so wird ihre Farbe immer schwächer und schliefslich scheiden sich aus der fast farblos gewordenen Lösung grofse, stark glänzende und vollkommen durchsichtige Krystalle ab, welche nahezu die Farbe des Nitroprussidnatriums besitzen. Meistens bestehen diese aus sāulenförmigen Prismen, die beim langsamen Verdunsten der Lösung sehr leicht in der Gröfse von mehreren Zollen erhalten werden können; einmal aber, als die Lösung nur eben den Boden des flachen Krystallisationsgefäfses bedeckte, erhielten wir grofse sechseckige Tafeln von derselben Farbe. In beiden Krystallformen ist das Salz wasserfrei und hat die von Rammelsberg *) gefundene Zusammensetzung.

0,5235 Grm. lieferten 0,13746 ans $=0,0869$ und 0,1476 $\mathrm{K}_{2} 8 \theta_{4}=0,18746 \mathrm{~K}$.

\begin{tabular}{|c|c|c|c|}
\hline \multirow[b]{2}{*}{ Mn } & \multicolumn{2}{|c|}{ Berechnet } & \multirow{2}{*}{$\begin{array}{c}\text { Gefunden } \\
16,60\end{array}$} \\
\hline & 55 & 16,75 & \\
\hline $3 \mathrm{~K}$ & 117,3 & 35,73 & 35,81 \\
\hline \multirow[t]{2}{*}{$6(\theta N)$} & 156 & 47,52 & - \\
\hline & 328,3 & 100,00 & \\
\hline
\end{tabular}

Das Salz ist demnach dem rothen Blutlaugensalz analog zusammengesetzt, mit welchem es auch isomorph ist, wie schon $\mathrm{Handl}^{* *}$ ) gefunden hat. Es entsteht aus dem Mangan-

*) Pogg. Ann. XLII, 117.

**) Jahresber. für Chemie u. s. w. f. 1859, 276. 
cyanür-Cyankalium, ebenso wie das rothe Blutlaugensalz aus dem gelben, durch 0xydation; während aber beim Ferrocyankalium ein starkes Oxydationsmittel erforderlich ist, genügt bei der entsprechenden Manganverbindung dazu schon der Sauerstoff der Luft. Schwieriger ist die momentane Bildung des rothen Salzes beim Kochen der wässerigen Lōsung des blauen Salzes zu erklären. Sehr wahrscheinlich findet dabei Zersetzung des Wassers statt. Die gleichzeitige Abscheidung von Manganoxydhydrat ist vielleicht die Folge einer secundären Reaction, denn auch das reine Salz scheidet beim Kochen seiner wässerigen Lösung Manganoxydhydrat ab, und wenn man das Erhitzen hinreichend lange fortsetzt und das verdampfende Wasser erneuert, bleibt keine Spur von Mangan gelöst. Aus diesem Grunde läfst sich das Salz auch nicht gut aus reinem Wasser umkrystallisiren. Die Gegenwart von Cyankalium macht das Salz beständiger.

Mit neutralen Metallsalzlōsungen giebt die frisch bereitete Lösung des rothen Salzes folgende Reactionen. Chlorbaryum, Chlorcalcium, schwefelsaure Magnesia, essigsaure Thonerde, salpetersaures Uranoxyd und Platinchlorid geben keine Niederschläge; Chlorkobult, schwefelsaures Nickel, Quecksilberchlorid und essigsaures BIei erzeugen braune Fällungen; schwefclsaures Kupfer giebt einen grauen, salpetersaures Silber einen gelblichbraunen, oxydfreier Eisenvitriol einen blauen, dem Berlinerblau ähnlichen Niederschlag; Eisenchlorid bewirkt keine Fällung, sondern giebt eine blaue Lösung, aus welcher sich nach längerem Stehen braune Flocken abscheiden. Zinnchlorür giebt einen weifsen, essigsaures Manganoxydul einen fleischrothen Niederschlag, der nach Rammelsberg dem Berlinerblau analog zusammengesetzt ist. Die meisten dieser Niederschläge sind, wie beim 
Cyanürsalz, nicht sehr beständig und lassen sich nicht ohne Zersetzung auswaschen.

Verdünnte Salzsäure und Salpetersäure erzeugen in der frisch bereiteten Lösung des rothen Salzes keine Fällung, wiewohl der Geruch nach Blausãure sehr deutlich auftritt. Schwefelalkalien fällen langsam Schwefelmangan.

\section{Mangancyanür-Cyannatrium.}

Versetzt man eine Lōsung von Cyannatrium (aus Blausäure und Natronlauge bereitet) tropfenweise mit essigsaurem Marigan, so treten genau dieselben Erscheinungen wie bei der Darstellung des Kaliumsalzes auf. Zuerst scheidet sich ein gelblich-weifser Körper ab, der beim Umschütteln wieder gelöst wird; dann fällt ein hlaugrüner, in überschüssigem Cyannatrium leicht löslicher Niederschlag. Diese Lösung scheidet, wenn sie concentrirt ist und namentlich wenn man sie auf Eis stellt, sehr bald grofse, durchsichtige, prachtvoll ausgebildete Octaëder von amethystrother Farbe ab. Die Krystallisationsfähigkeit dieses Salzes ist sehr grofs. Versetzt man die Lösung mit Alkohol, bis eine bleibende Trübung eintritt, so krystallisirt das Salz in langen, ebenso gefärbten Spiersen.

Die Krystalle verwittern noch rascher, als die der Kaliumverbindung, werden violettblau und verlieren ihre Durchsichtigkeit. Die Krystallwasserbestimmung wurde in ähnlicher Weise wie beim Kaliumsalz ausgeführt, das Trocknen im Luftstrome von 30 bis $40^{\circ}$ aber nur so lange fortgesetzt, bis einige Krystalle anfingen ihre Farbe zu ändern. Dann wurden möglichst schnell die noch unveränderten herausgesucht, gewogen und auf $100^{\circ}$ erhitzt. In beiden Formen enthielt das Salz dieselbe Menge Krystallwasser.

I. 0,6645 Grm. der octaëdriscben Krystalle verloren $0,2125 \mathbf{H}_{8} \Theta$. 
II. 0,823 Grm. der mit Alkohol erhaltenen Spiefse verloren 0,26275 $\mathrm{H}_{2} \mathrm{\theta}$.

III. 0,14512 Grm. des wasserfreien Salzes gaben 0,0413 Mns = $0,0261 \mathrm{Mn}$ und $0,13668 \mathrm{Na}_{2} \mathrm{~S} \theta_{4}=0,04427 \mathrm{Na}$.

IV. $0,6843 \mathrm{Grm}$. gaben $0,19278 \mathrm{MnS}=0,12187$ Mn und 0,64659 $\mathrm{Na}_{2} \mathrm{~S} \theta_{4}=0,20946 \mathrm{Na}$.

V. $0,5215 \mathrm{Grm}$. gaben $0,14653 \mathrm{MnS}=0,09241 \mathrm{Mn}$ und 0,4918 $\mathrm{Na}_{2} \mathrm{SO}_{1}=0,15932 \mathrm{Na}$.

\begin{tabular}{|c|c|c|c|c|c|c|c|}
\hline & \multicolumn{3}{|c|}{ Berechnet } & \multicolumn{4}{|c|}{ Gefunden } \\
\hline $\mathbf{M n}$ & 55 & \multicolumn{2}{|c|}{18,15} & $\begin{array}{r}\text { III. } \\
17,99\end{array}$ & \multicolumn{2}{|c|}{ IV. } & $\begin{array}{c}V \\
17,72\end{array}$ \\
\hline $4 \mathrm{Na}$ & 92 & \multicolumn{2}{|c|}{30,36} & 30,50 & \multicolumn{2}{|c|}{30,61} & 30,55 \\
\hline \multirow[t]{4}{*}{$6(\mathrm{GN})$} & 156 & \multicolumn{2}{|c|}{51,49} & - & \multicolumn{2}{|c|}{-} & - \\
\hline & 303 & \multicolumn{2}{|c|}{100,00} & & & & \\
\hline & & \multicolumn{2}{|c|}{ Berechnet } & \multicolumn{4}{|c|}{ Gefunden } \\
\hline & & & & & & & I. \\
\hline \multirow[t]{3}{*}{$\mathrm{Cy}_{2}+$} & $4 \mathrm{NaCy}$ & $\mathbf{3 0 3}$ & 67,78 & & & & - \\
\hline & $3 \mathrm{H}_{8} \Theta$ & 144 & 32,22 & & & &, 92 \\
\hline & & 447 & 100,00 & & & & \\
\hline
\end{tabular}

Die Zusammensetzung des Mangancyanür-Cyannatriums läfst sich demnach durch die Formel $\mathrm{AnCy}_{2}+4 \mathrm{NaCy}+8 \mathrm{H}_{2} \mathrm{\theta}$ ausdrücken. Es enthält $4 \mathrm{Mol}$. Krystallwasser weniger als das analoge Eisencyanür-Cyannatrium.

In kaltem Wasser löst es sich leicht zu einer klaren, schwach gelben Lösung, welche sich nach einiger Zeit trübt und allmälig einen blaugrünen Niederschlag absetzt. Es verhält sich das Salz also genau so, wie die entsprechende Kaliumverbindung, und der grüne Niederschlag ist unzweifelhaft nach der Formel $\mathrm{HnCy}_{2}+\mathrm{NaCy}$ zusammengesetzt. Wir hielten es für überflüssig, denselben zu analysiren.

\section{Mangancyanid-Cyannatrium.}

Wir erhielten dieses Salz genau auf dieselbe Weise, wie die entsprechende Kaliumverbindung. Das amethystrothe $\mathrm{Cy}-$ anürsalz geht sowohl beim Verweilen in der Mutterlauge, wie beim Kochen seiner Lösung eben so leicht und unter 
denselben Erscheinungen in das Cyanidsalz über, wie das blaue Mangancyanür-Cyankalium.

Das Mangancyanid-Cyannatrium krystallisirt aus einer Lōsung, die freies Cyannatrium enthält, entweder in Octaëdern, die bei auffallendem Licht beinahe schwarz, bei durchfallendem blafsviolett sind, oder in rothen Prismen, die dem rothen Kaliumsalze sehr ähnlich aussehen.

Die verschiedene Form hängt von einem verschiedenen Krystallwassergehalt ab; aber beide Arten von Krystallen verwittern so leicht, dafs uns trotz aller Vorsicht eine genaue Bestimmung des Wassers nicht gelang.

I. $0,452 \mathrm{Grm}$. der schwarzen Krystalle verloren bei $100^{\circ} 0,09643$ $\mathrm{H}_{2} \Theta=21,16 \mathrm{pC}$.

Die Formel $\mathrm{MnCy}_{8}+3 \mathrm{NaCy}+4 \mathrm{H}_{2} \theta$ verlangt 20,45 pC., die Formel $\mathrm{MnCy}_{3}+3 \mathrm{NaCy}+4_{12}^{1 / \mathrm{H}_{2}} \mathrm{O} 22,43$ pC. $\mathrm{H}_{2} \theta$.

II. $0,1615 \mathrm{Grm}$. der rothen Krystalle verloren bei $100^{\circ} 0,02025 \mathrm{H}_{2} \theta$ $=12,53 \mathrm{pC}$.

Die Formel $\mathrm{NCy}_{3}+3 \mathrm{NaCy}+2 \mathrm{H}_{2} \theta$ verlangt 11,39 pC., die Formel $\mathrm{MnCy}_{8}+3 \mathrm{NaCy}+2_{12}^{1 / 2} \mathrm{H}_{2} \Theta 13,84 \mathrm{pC}$.

Diese Bestimmungen, obwohl sie nicht sehr genau sind machen es doch wahrscheinlich, dafs das dunkele octaëdrische Salz 4 Mol., das rothe dagegen nur 2 Mol. Krystallwasser enthält.

Es war uns unmöglich, die Verhältnisse zu präcisiren, unter welchen sich eine der beiden Krystallformen bildet; denn wir erbielten bei mehrmaligem Umkrystallisiren desselben Salzes, unter anscheinend denselben Verhältnissen, bald die eine, bald die andere Form.

Gegen Wasser verhält sich das Salz genau so, wie die entsprechende Kaliumverbindung.

Die Analyse des wasserfreien Salzes ergab folgende Zablen :

0,4281 Grm. lieferten 0,13165 Yns $=0,08321$ und $0,3281 \mathrm{Na}_{2} 8 \theta_{4}=0,10629 \mathrm{Na}$. 


\begin{tabular}{lrrc} 
& \multicolumn{2}{c}{ Berechnet } & Gefunden \\
\cline { 2 - 3 } $3 \mathrm{Na}$ & 55 & 19,64 & 19,44 \\
$\mathbf{3 N}$ & 24,64 & 24,83 \\
$6(\mathrm{GN})$ & 156 & 55,72 & -
\end{tabular}

\section{v. Mangancyanïr-Cyanammonium.}

Fügt man zu einer (aus Ammoniak und wässeriger Blausäure bereiteten) Lōsung von Cyanammonium tropfenweise essigsaures Mangan, so fällt zuerst ein ganz weifser krystallinischer Niederschlag, der sich beim Umschütteln wieder auflöst, wenn die Cyanammoniumlösung concentrirt ist. Fügt man mehr Manganlösung hinzu, so fällt ein grūnlicher Niederschlag, der sich auswaschen und neben Schwefelsäure trocknen lāfst, aber bei $100^{\circ}$ zersetzt wird. Die Analyse (s. unten I und II) zeigte, dafs er nach der Formel $\mathrm{MnCy}_{2}+\mathrm{NH}_{4} \mathrm{Cy}$, also den bei Anwendung von Cyankalium oder Cyannatrium entstehenden grünen Niederschlägen völlig analog zusammengesetzt ist. In überschüssigem concentrirtem Cyanammonium löst er sich fast vollständig $\mathrm{zu}$ einer gelben Lösung auf. Diese enthält unzweifelhaft ein Salz $\mathrm{MnCy}_{2}+4 \mathrm{NH}_{4} \mathrm{Cy}$, welches wir aber nicht daraus ahzuscheiden vermochten. Setzt man die Lösung an einen kühlen Ort, so scheidet sich, selbst wenn ziemlich viel überschüssiges Cyanammonium vorhanden ist, doch nach einiger Zeit der unveränderte grünliche Niederschlag wieder ab. Alkohol erzeugt in der frisch bereiteten klaren Lösung sofort eine starke Fällung, aber der Niederschlag ist ebenfalls nichts Anderes, als das grüne Salz (s. unten Analyse III). Beim Verdunsten der frisch bereiteten Lösung wird derselbe Körper (Analyse IV) abgeschieden. Dabei färbt sich die Flüssigkeit zwar roth, aber sie enthält nur eine kaum noch nachweisbare Spur von Mangan. 
Das Mangancyanür-Cyanammonium unterscheidet sich demnach durch seine leichtere Zersetzbarkeit wesentlich von den entsprechenden Kalium- und Natriumverbindungen. Es erleidet auch bei Gegenwart von überschüssigem Cyanammonium dieselben Zersetzungen, welche jene Verbindungen nur in Berührung mit reinem Wasser zeigen.

I. 0,3321 Grm. gaben 0,19227 $\mathrm{ng}=0,12154 \mathrm{Mn}$.

II. 0,1236 Grm. gaben mit Natronkalk geglüht $0,05536 \quad \mathrm{NH}_{3}=$ $0,04559 \mathrm{~N}$.

III. 0,13485 Grm. gaben 0,07004 $\mathrm{MnS}=0,04428 \mathrm{Mn}$.

IV. 0,4214 Grm. gaben 0,24165 Hns $=0,15288$ m.

\begin{tabular}{lrrrrrrr} 
& \multicolumn{3}{c}{ Berechnet } & & \multicolumn{3}{c}{ Gefunden } \\
Mn & 55 & 36,42 & & II. & III. & IV. \\
$4 \mathrm{H}$ & 4 & 2,65 & & - & - & - & - \\
36 & 36 & 23,84 & & - & - & - & - \\
$4 \mathrm{~N}$ & 56 & 37,09 & & - & 36,88 & - & -
\end{tabular}

VI. Mangancyanür-Cyanbaryum.

Beim Vermischen einer concentrirten Lösung von Cyanbaryum (durch Auflösen von festem Barythydrat in concentrirter Blausäure bereitet) mit essigsaurem Mangan erhält man unter ähnlichen Erscheinungen, wie bei den vorher beschriebenen Salzen, eine gelbe Lösung, aus welcher sich beim Abkühlen kleine, rein blaue, zu concentrischen Gruppen vereinigte Krystalle abscheiden. Um das Salz rein und frei von kohlensaurem Baryum zu erhalten, mufs man es in einem kohlensäurefreien Raume krystallisiren lassen. Es ist beständiger, als die Alkalisalze. Kaltes Wasser löst und zersetzt es nur langsam. Es enthält Krystallwasser, welches über Schwefelsäure langsam, bei $100^{\circ}$ rasch entweicht. Das bei $100^{\circ}$ getrocknete Salz hat die Zusammensetzung $\mathrm{HnCy}_{2}+2 \mathrm{BaCy}_{2}$. Zur Analyse wurde es in heifser verdünnter Salzsäure 
gelöst, das Baryum mit Schwefelsãure und aus dem Filtrate das Mangan mit Ammoniak und Schwefelammonium gefällt.

0,5133 Grm. gaben $0,0900 \mathrm{HB}=0,05691$ Mnd $0,4960 \mathrm{BaSO}_{4}$ $=0,2916 \mathrm{Ba}$.

\begin{tabular}{|c|c|c|c|}
\hline \multirow[b]{2}{*}{$M n$} & \multicolumn{2}{|c|}{ Berechnet } & \multirow{2}{*}{$\begin{array}{c}\text { Gefunden } \\
11,09\end{array}$} \\
\hline & 55 & 11,34 & \\
\hline $2 \mathrm{Ba}$ & 274 & 56,50 & 56,80 \\
\hline $6(\mathrm{GN})$ & 156 & 32,16 & - \\
\hline & 485 & 100,00 . & \\
\hline
\end{tabular}

VII. Mangancyanid-Cyanbaryum.

Bei längerem Verweilen in der Mutterlauge geht das Mangancyanür-Cyanbaryum eben so, wie die Alkalisalze, in diese Verbindung über und die Umwandlung erfolgt auch hier rascher beim Erwärmen. Dampft man die gelbe Lõsung im Wasserbade $a b$, so bleibt eine rothe Masse zurũck, die sich zum gröfsten Theile in Wasser mit rother Farbe auflōst. Diese Lösung scheidet beim freiwilligen Verdunsten neben Schwefelsäure und Aetzkalk eine hellrothe krystallinische Masse ab, aus der wir auch durch nochmaliges Lösen in cyanbaryumhaltigem Wasser und freiwilliges Verdunsten keine regelmăfsigen Krystalle erhalten konnten.

Das neben Schwefelsäure getrocknete Salz ist wasserfrei und nach der Formel $2\left(\mathrm{MnCy}_{3}\right)+3\left(\mathrm{BaCy}_{2}\right)$ zusammengesetzt.

0,63425 Grm. gaben 0,10254 Kng $=0,08315$ Mn und 0,53439 $\mathrm{BaSO}_{4}=0,31421 \mathrm{Ba}$.

\begin{tabular}{|c|c|c|c|}
\hline & \multicolumn{2}{|c|}{ Berechnet } & Gefunden \\
\hline $2 \mathrm{Mnn}$ & 110 & 13,21 & 13,11 \\
\hline $3 \mathbf{B a}$ & 411 & 49,34 & 49,52 \\
\hline $12(\mathrm{GN})$ & 312 & 37,45 & - \\
\hline & 833 & 100,00 & \\
\hline
\end{tabular}


VIII. Manguncyanür-Cyancalcium.

Wir erhielten dieses Salz durch Vermischen von essigsaurem Mangan mit einem geringen Ueberschufs einer frisch bereiteten Lösung von Cyancalcium und Ausfällen mit Alkohol. Es schied sich als ein krystallinischer blauer Niederschlag ab. Zur Analyse wurde das getrocknete Salz in Salzsãure gelōst, die Lösung mit essigsaurem Natron versetzt, mit Chlorgas gesältigt und das Mangan durch einen kleinen Ueberschurs von Ammoniak gefält. Nach dem Verdunsten des überschüssigen Ammoniaks wurde der Nirderschlag abfiltrirt, geglüht und gewogen. Aus dem Filtrat wurde das Calcium auf gewöhnliche Weise mit oxalsaurem Ammonium gefältt.

0,64025 Grm. gaben $0,16607 \mathrm{Mn}_{3} \theta_{4}=0,11966 \mathrm{Mn}$ und $0,24839 \mathrm{Ga} \theta$ $=0,17725 \mathrm{Ga}$.

\begin{tabular}{crcc} 
& \multicolumn{2}{c}{ Berechnet } & Gefunden \\
\cline { 2 - 4 } $2 \mathrm{Fu}$ & 55 & 18,90 & 18,68 \\
$2 \mathrm{Ga}$ & 80 & 27,49 & 27,70 \\
$6(G N)$ & 156 & 58,61 & -
\end{tabular}

Das Salz hat demnach die Zusammensetzung MnG $\mathrm{Ay}_{2}$ $+2 \mathrm{GaCy}_{2}$. Es verhält sich genau wie das Baryumsalz und geht beim Verdunsten seiner mit überschüssigem Cyancalcium versetzten Lösung in einer kohlensäurefreien Atmosphäre in das Cyanidsalz $2\left(\mathrm{AnCy}_{3}\right)+3\left(\mathrm{GaCy}_{2}\right)$ über, welches wir indefs nicht in gut ausgebildeten Krystallen, sondern nur als hellrothe krystallinische Masse erhalten kunnten.

0,97362 Grm. des wasserfreien Salzes gaben $0,2686 \quad \mathrm{nn}_{9} \theta_{4}=$ 0,19354 and $0,74806 \mathrm{GaS}_{4}=0,22001 \mathrm{Ga}$.

\begin{tabular}{|c|c|c|c|}
\hline \multirow[b]{2}{*}{$2 \mathrm{Mn}$} & \multicolumn{2}{|c|}{ Berechnet } & \multirow{2}{*}{$\begin{array}{c}\text { Gefunden } \\
19,88\end{array}$} \\
\hline & 110 & $\overline{20,29}$ & \\
\hline $36 \mathbf{a}$ & 120 & 22,14 & 22,60 \\
\hline $12(G N)$ & 312 & 57,57 & - \\
\hline
\end{tabular}


Diese Versuche zeigen, dafs das Mangan sich bei der Bildung von Doppelcyaniden vollständig analog dem Eisen verhält. Alle Verbindungen sind nach denselben allgemeinen Forıneln wie die Eisenverbindungen zusammengesetzt. In Betreff der Eigenschaften tritt indefs eine nicht unwesentliche Verschiedenheit hervor; denn während die Eisendoppelcyanide sehr feste und beständige Verbindungen sind, ist bei den entsprechenden Manganverbindungen, die sich sämmtlich durch ihre prachtvollen Farben so sehr auszeichnen, gerade die aufserordentlich leichte Zersetzbarkeit ein characteristisches Merkmal.

Göttingen, den 12. August 1867.

Ueber das intermediäre Anhydrid von Kieselsäure und Essigsäure;

von C. Friedel und A. Ladenburg*).

Gerhard t's Entdeckung der Anhydride einbasischer Säuren hat bis jetzt noch nicht zu der von gemischten Anhydriden mehrbasischer Säuren mit ein- oder mehrbasischen Säuren geführt; wir meinen solche, in denen kein typischer Wasserstoff mehr vorhanden, sondern derselbe vollständig gegen Säureradicale ausgetauscht ist.

Freilich sind mehrere Reactionen bekannt, in denen derartige Körper sich hätten bilden können; doch ist es den Autoren niemals gelungen, die Verbindungen zu isoliren. So haben Kämmerer und Carius im Jahre 1864**) Chlor-

*) Der franz. Acad. den 14. Januar 1867 mitgetheilt.

**) S. diese Annalen CXXXI, 153. 